\title{
MODIFIED QUADRATIC RESIDUE CONSTRUCTIONS AND NEW EXTREMAL BINARY SELF-DUAL CODES OF LENGTHS 64, 66 AND 68
}

\author{
JOE GILDEA, HOLLY HAMILTON, ABIDIN KAYA AND BAHATTIN YILDIZ
}

\begin{abstract}
In this work we consider modified versions of quadratic double circulant and quadratic bordered double circulant constructions over the binary field and the rings $\mathbb{F}_{2}+u \mathbb{F}_{2}$ and $\mathbb{F}_{4}+u \mathbb{F}_{4}$ for different prime values of $p$. Using these constructions with extensions and neighbors we are able to construct a number of extremal binary self-dual codes of different lengths with new parameters in their weight enumerators. In particular we construct 2 new codes of length 64,4 new codes of length 66 and 14 new codes of length 68 . The binary generator matrices of the new codes are available online at $[8]$.
\end{abstract}

\section{INTRODUCTION}

Self-dual codes are a special class of codes that have algebraic and combinatorial properties, which connect them to many different structures such as lattices, invariant theory, designs, cryptography and recently with quantum codes ([11]). There are many different approaches of constructing selfdual codes in the literature. One particular construction makes use of quadratic residues modulo a prime $p$.

Let $R$ be a finite commutative Frobenius ring of characteristic 2 and $p$ be prime. Let $\gamma_{i} \in R$, $A$ be a $p \times p$ circulant matrix, $Q_{r}(a, b, c)$ be the $p \times p$ circulant matrix with three free variables, obtained through the quadratic residues and non-residues modulo $p$. Thus, the first row of $\bar{r}=$ $\left(r_{0}, r_{1}, \ldots, r_{p-1}\right)$ of $Q_{p}(a, b, c)$ is determined by the following rule:

$$
r_{i}= \begin{cases}a & \text { if } i=0 \\ b & \text { if } i \text { is a quadratic residue modulo } p \\ c & \text { if } i \text { is a quadratic non-residue modulo } p .\end{cases}
$$

This type of quadratic circulant matrix and a bordered version were first introduced by Gaborit in [7] to construct self-dual codes over fields and later were extended over rings in [13] and [16] to construct self-dual codes over different alphabets. The main construction used in the works mentioned was to take a matrix of the form $\left[I_{p} \mid A\right]$ where $A$ is either $Q_{p}(a, b, c)$ or its bordered version. Note that the quadratic circulant matrix is a special case of a double circulant matrix which produces self-dual quasi-cyclic codes ([20]).

In this work, we modify this construction by replacing $I_{p}$ with an arbitrary circulant matrix in the double circulant case, and we do a similar replacement in the bordered case. More precisely, we consider the following type of matrices:

$$
M=\left(Q_{p}(a, b, c) \mid A\right),
$$

where $A$ is a $p \times p$ circulant matrix and

$$
M=\left(\begin{array}{c|ccc|c|ccc}
\gamma_{1} & \gamma_{2} & \cdots & \gamma_{2} & \gamma_{3} & \gamma_{4} & \cdots & \gamma_{4} \\
\hline \gamma_{2} & & & & \gamma_{4} & & & \\
\vdots & & Q_{p}(a, b, c) & & \vdots & & A & \\
\gamma_{2} & & & & \gamma_{4} & & &
\end{array}\right),
$$

where $A$ is a $p \times p$ circulant matrix.

1991 Mathematics Subject Classification. 94B05, 15B33.

Key words and phrases. combinatorial problems; extremal self-dual codes; codes over rings; quadratic residues; quadratic circulant matrices. 
We consider these constructions for different prime values $p$ and over the binary field as well as the ring extensions $\mathbb{F}_{2}+u \mathbb{F}_{2}$ and $\mathbb{F}_{4}+u \mathbb{F}_{4}$. This is a novel construction method for self-dual codes, that combines several approaches in the literature. The strength and novelty of the constructions are demonstrated by the numerous new extremal self-dual codes that we are able to obtain using these methods. In particular, we are able to construct 14 new extremal binary self-dual codes of length 68, 4 new extremal binary self-dual codes of length 66 and 2 new extremal binary self-dual codes of length 64 . The new beta values and the weight enumerator classes of the codes are given in section 4 .

The rest of the work is organized as follows. In section 2, we give some background on self-dual codes, and the different alphabets that we use. In section 3 we give the constructions. In section 4 , we give the numerical results obtained from the constructions. We finish by recapitulating the results and pointing out possible directions for future research.

\section{Preliminaries}

Let $R$ be a commutative Frobenius ring of characteristic 2. A code $C$ of length $n$ over $R$ is an $R$-submodule of $R^{n}$. Elements of the code $C$ are called codewords of $C$. Let $x=\left(x_{1}, x_{2}, \ldots, x_{n}\right)$ and $y=\left(y_{1}, y_{2}, \ldots, y_{n}\right)$ be two elements of $R^{n}$. The duality is understood in terms of the Euclidean inner product; $\langle x, y\rangle_{E}=\sum x_{i} y_{i}$. The dual $C^{\perp}$ of the code $C$ is defined as

$$
C^{\perp}=\left\{x \in R^{n} \mid\langle x, y\rangle_{E}=0 \text { for all } y \in C\right\} .
$$

We say that $C$ is self-dual if $C=C^{\perp}$. Two self-dual binary codes of dimension $k$ are said to be neighbors if their intersection has dimension $k-1$. A binary self-dual code is called Type II (or doubly even) if the weights of all codewords are divisible by 4, otherwise it is called Type I (or singly even). The best upper bound on the minimum Hamming distance of a binary self-dual code was proved in [19].

Theorem 2.1. ([19]) Let $d_{I}(n)$ and $d_{I I}(n)$ be the minimum distance of a Type I and Type II binary code of length $n$, respectively. Then

$$
d_{I I}(n) \leq 4\left\lfloor\frac{n}{24}\right\rfloor+4
$$

and

$$
d_{I}(n) \leq \begin{cases}4\left\lfloor\frac{n}{24}\right\rfloor+4 & \text { if } n \not \equiv 22 \quad(\bmod 24) \\ 4\left\lfloor\frac{n}{24}\right\rfloor+6 & \text { if } n \equiv 22 \quad(\bmod 24)\end{cases}
$$

Self-dual codes meeting these bounds are called extremal.

2.1. The ambient rings. While all of the theoretical results we obtain throughout the paper are valid for all Frobenius rings of characteristic 2, we will be focusing mainly on the binary field $\mathbb{F}_{2}$ and the rings $\mathbb{F}_{2}+u \mathbb{F}_{2}, \mathbb{F}_{4}+u \mathbb{F}_{4}$ for computational purposes. Similar rings have been considered in coding theory in many different works, e.g. [21] and [22].

Let $\mathbb{F}_{4}=\mathbb{F}_{2}(\omega)$ be the quadratic field extension of the binary field $\mathbb{F}_{2}=\{0,1\}$, where $\omega^{2}+\omega+1=$ 0 . The ring $\mathbb{F}_{4}+u \mathbb{F}_{4}$ defined via $u^{2}=0$ is a commutative binary ring of size 16 . We may easily observe that it is isomorphic to $\mathbb{F}_{2}[\omega, u] /\left\langle u^{2}, \omega^{2}+\omega+1\right\rangle$. The ring has a unique non-trivial ideal $\langle u\rangle=\{0, u, u \omega, u+u \omega\}$. Note that $\mathbb{F}_{4}+u \mathbb{F}_{4}$ can be viewed as an extension of $\mathbb{F}_{2}+u \mathbb{F}_{2}$ and so we can describe any element of $\mathbb{F}_{4}+u \mathbb{F}_{4}$ in the form $\omega a+\bar{\omega} b$ uniquely, where $a, b \in \mathbb{F}_{2}+u \mathbb{F}_{2}$.

$$
\begin{array}{lcl}
\left(\mathbb{F}_{4}+u \mathbb{F}_{4}\right)^{n} & \psi_{\mathbb{F}_{4}+u \mathbb{F}_{4}} & \left(\mathbb{F}_{2}+u \mathbb{F}_{2}\right)^{2 n} \\
& & \downarrow \varphi_{\mathbb{F}_{2}+u \mathbb{F}_{2}} \\
\mathbb{F}_{4}^{2 n}+u \mathbb{F}_{4} & \psi_{\mathbb{F}_{4}} & \mathbb{F}_{2}^{4 n} \\
\hline
\end{array}
$$

Let us recall the following Gray Maps from [7,18] and [6];

$$
\begin{aligned}
\psi_{\mathbb{F}_{4}} & : a \omega+b \bar{\omega} \mapsto(a, b), \quad a, b \in \mathbb{F}_{2}^{n} \\
\varphi_{\mathbb{F}_{2}+u \mathbb{F}_{2}} & : a+b u \mapsto(b, a+b), \quad a, b \in \mathbb{F}_{2}^{n} \\
\psi_{\mathbb{F}_{4}+u \mathbb{F}_{4}} & : a \omega+b \bar{\omega} \mapsto(a, b), \quad a, b \in\left(\mathbb{F}_{2}+u \mathbb{F}_{2}\right)^{n} \\
\varphi_{\mathbb{F}_{4}+u \mathbb{F}_{4}} & : a+b u \mapsto(b, a+b), \quad a, b \in \mathbb{F}_{4}^{n}
\end{aligned}
$$


Note that these Gray maps preserve orthogonality in the respective alphabets, for the details we refer to [18]. The binary codes $\varphi_{\mathbb{F}_{2}+u \mathbb{F}_{2}} \circ \psi_{\mathbb{F}_{4}+u \mathbb{F}_{4}}(C)$ and $\psi_{\mathbb{F}_{4}} \circ \varphi_{\mathbb{F}_{4}+u \mathbb{F}_{4}}(C)$ are equivalent to each other. The Lee weight of an element in $\mathbb{F}_{4}+u \mathbb{F}_{4}$ is defined to be the Hamming weight of its binary image under any of the previously mentioned compositions of the maps. A self-dual code is said to be of Type II if the Lee weights of all codewords are multiples of 4, otherwise it is said to be of Type I.

Proposition 2.2. ([18]) Let $C$ be a code over $\mathbb{F}_{4}+u \mathbb{F}_{4}$. If $C$ is self-orthogonal, so are $\psi_{\mathbb{F}_{4}+u \mathbb{F}_{4}}(C)$ and $\varphi_{\mathbb{F}_{4}+u \mathbb{F}_{4}}(C)$. C is a Type $I$ (resp. Type II) code over $\mathbb{F}_{4}+u \mathbb{F}_{4}$ if and only if $\varphi_{\mathbb{F}_{4}+u \mathbb{F}_{4}}(C)$ is a Type $I$ (resp. Type II) $\mathbb{F}_{4}$-code, if and only if $\psi_{\mathbb{F}_{4}+u \mathbb{F}_{4}}(C)$ is a Type $I$ (resp. Type II) $\mathbb{F}_{2}+u \mathbb{F}_{2}$-code. Furthermore, the minimum Lee weight of $C$ is the same as the minimum Lee weight of $\psi_{\mathbb{F}_{4}+u \mathbb{F}_{4}}(C)$ and $\varphi_{\mathbb{F}_{4}+u \mathbb{F}_{4}}(C)$.

Corollary 2.3. Suppose that $C$ is a self-dual code over $\mathbb{F}_{4}+u \mathbb{F}_{4}$ of length $n$ and minimum Lee distance d. Then $\varphi_{\mathbb{F}_{2}+u \mathbb{F}_{2}} \circ \psi_{\mathbb{F}_{4}+u \mathbb{F}_{4}}(C)$ is a binary $[4 n, 2 n, d]$ self-dual code. Moreover, $C$ and $\varphi_{\mathbb{F}_{2}+u \mathbb{F}_{2}} \circ \psi_{\mathbb{F}_{4}+u \mathbb{F}_{4}}(C)$ have the same weight enumerator. If $C$ is Type I (Type II), then so is $\varphi_{\mathbb{F}_{2}+u \mathbb{F}_{2}} \circ \psi_{\mathbb{F}_{4}+u \mathbb{F}_{4}}(C)$.

In subsequent sections we will be writing tables in which vectors with elements from the rings $\mathbb{F}_{2}+u \mathbb{F}_{2}$ and $\mathbb{F}_{4}+u \mathbb{F}_{4}$ will appear. In order to avoid writing long vectors with elements that can be confused with other elements, we will be describing the elements of this ring in a shorthand way, which will make the tables more compact. For the elements of $\mathbb{F}_{2}+u \mathbb{F}_{2}$ we will use $0 \rightarrow 0,1 \rightarrow 1, u \rightarrow u$ and $1+u \rightarrow 3$.

For the elements of $\mathbb{F}_{4}+u \mathbb{F}_{4}$, we use the ordered basis $\{u \omega, \omega, u, 1\}$ to express the elements of $\mathbb{F}_{4}+u \mathbb{F}_{4}$ as binary strings of length 4 . Then we will use the hexadecimal number system to describe each element:

$0 \leftrightarrow 0000,1 \leftrightarrow 0001,2 \leftrightarrow 0010,3 \leftrightarrow 0011,4 \leftrightarrow 0100,5 \leftrightarrow 0101,6 \leftrightarrow 0110,7 \leftrightarrow 0111,8 \leftrightarrow 1000$, $9 \leftrightarrow 1001, A \leftrightarrow 1010, B \leftrightarrow 1011, C \leftrightarrow 1100, D \leftrightarrow 1101, E \leftrightarrow 1110, F \leftrightarrow 1111$.

For example $1+u \omega$ corresponds to 1001, which is represented by the hexadecimal 9 , while $\omega+u \omega$ corresponds to 1100 , which is represented by $C$.

\section{The Constructions}

We will discuss the constructions in two classes. The first will be the pure double circulant and the second will be the bordered one.

3.1. Generalized quadratic double circulant. Define the matrix $M=\left(Q_{p}(a, b, c) \mid A\right)$ where $A$ is a $p \times p$ circulant matrix. Let $C$ be the linear code of length $2 p$, generated by the matrix $M$. Then

$$
M M^{T}=Q_{p}(a, b, c) Q_{p}(a, b, c)^{T}+A A^{T} .
$$

Note that $Q_{p}(a, b, c) Q_{p}(a, b, c)^{T}$ has previously been calculated in [7] where $Q_{p}(a, b, c)$ is a $p \times p$ circulant over a finite field. When $Q_{p}(a, b, c)$ is a $p \times p$ circulant over a ring of characterisitc 2 , we can see that

$Q_{p}(a, b, c) Q_{p}(a, b, c)^{T}= \begin{cases}Q_{p}\left(a^{2}, b^{2}+k(b+c)^{2}, c^{2}+k(b+c)^{2}\right) & \text { if } p=4 k+1 \\ Q_{p}\left(a^{2}+b^{2}+c^{2}, a b+a c+k\left(b^{2}+c^{2}\right)+b c, a b+a c+k\left(b^{2}+c^{2}\right)+b c\right) & \text { if } p=4 k+3\end{cases}$

Combining this with (1), and considering the characteristic we get the following main result:

Theorem 3.1. If $C$ is a linear code generated by $M$ over a ring of characteristic 2, then $C$ is a self-orthogonal code if and only if:

$$
A A^{T}=\left\{\begin{array}{ll}
Q_{p}\left(a^{2}, b^{2}+k(b+c)^{2}, c^{2}+k(b+c)^{2}\right) & \text { if } p=4 k+1 \\
Q_{p}\left(a^{2}+b^{2}+c^{2}, a b+a c+k\left(b^{2}+c^{2}\right)+b c, a b+a c+k\left(b^{2}+c^{2}\right)+b c\right) & \text { if } p=4 k+3
\end{array} .\right.
$$


3.2. Generalized bordered quadratic residue construction. We will now define a bordered version of the construction. Define the following matrix:

$$
M=\left(\begin{array}{c|ccc|c|ccc}
\gamma_{1} & \gamma_{2} & \ldots & \gamma_{2} & \gamma_{3} & \gamma_{4} & \cdots & \gamma_{4} \\
\hline \gamma_{2} & & & & \gamma_{4} & & & \\
\vdots & & Q_{p}(a, b, c) & & \vdots & & A & \\
\gamma_{2} & & & & \gamma_{4} & & &
\end{array}\right),
$$

where $\gamma_{i}$ are some elements from the ambient ring $R$, which we assume to be a ring of characteristic 2 (including the binary field).

Let $C$ be a code that is generated by the matrix $M$ over $R$. Then, the code $C$ has length $2 p+2$.

To make the computations easier, we let $M=\left(\begin{array}{cccc}B_{1} & B_{2} & B_{3} & B_{4} \\ B_{2}^{T} & Q & B_{4}^{T} & A\end{array}\right)$ where $B_{1}=\left(\gamma_{1}\right), B_{2}=$ $\left(\gamma_{2}, \ldots, \gamma_{2}\right), B_{3}=\left(\gamma_{3}\right), B_{4}=\left(\gamma_{4}, \ldots, \gamma_{4}\right)$ and $Q=Q_{p}(a, b, c)$. Then,

$$
M M^{T}=\left(\begin{array}{cc}
B_{1} B_{1}^{T}+B_{2} B_{2}^{T}+B_{3} B_{3}^{T}+B_{4} B_{4}^{T} & B_{1} B_{2}+B_{2} Q^{T}+B_{3} B_{4}+B_{4} A^{T} \\
B_{2}^{T} B_{1}^{T}+Q B_{2}^{T}+B_{4}^{T} B_{3}^{T}+A B_{4}^{T} & B_{2}^{T} B_{2}+Q Q^{T}+B_{4}^{T} B_{4}+A A^{T}
\end{array}\right)
$$

where

- $B_{1} B_{1}^{T}+B_{2} B_{2}^{T}+B_{3} B_{3}^{T}+B_{4} B_{4}^{T}=\gamma_{1}^{2}+\left(\gamma_{2}, \ldots, \gamma_{2}\right)\left(\gamma_{2}, \ldots, \gamma_{2}\right)^{T}+\gamma_{3}^{2}+\left(\gamma_{4}, \ldots, \gamma_{4}\right)\left(\gamma_{4}, \ldots, \gamma_{4}\right)^{T}$

$$
\begin{aligned}
& =\gamma_{1}^{2}+p \gamma_{2}^{2}+\gamma_{3}^{2}+p \gamma_{4}^{2} \\
& =\left(\sum_{i=1}^{4} \gamma_{i}\right)^{2} .
\end{aligned}
$$

- $B_{1} B_{2}+B_{2} Q^{T}+B_{3} B_{4}+B_{4} A^{T}=\gamma_{1}\left(\gamma_{2}, \ldots, \gamma_{2}\right)+\left(\gamma_{2}, \ldots, \gamma_{2}\right) Q^{T}+\gamma_{3}\left(\gamma_{4}, \ldots, \gamma_{4}\right)+\left(\gamma_{4}, \ldots, \gamma_{4}\right) A^{T}$

$$
\begin{aligned}
& \quad=\left(\gamma_{1} \gamma_{2}, \ldots, \gamma_{1} \gamma_{2}\right)+\left(\gamma_{2}\left(a+b \lambda_{p}+c \lambda_{p}\right), \ldots, \gamma_{2}\left(a+b \lambda_{p}+c \lambda_{p}\right)\right) \\
& \quad+\left(\gamma_{3} \gamma_{4}, \ldots, \gamma_{3} \gamma_{4}\right)+\left(\gamma_{4} \mu, \ldots, \gamma_{4} \mu\right) \\
& =\left(\gamma_{1} \gamma_{2}+\gamma_{2}\left(a+b \lambda_{p}+c \lambda_{p}\right)+\gamma_{3} \gamma_{4}+\gamma_{4} \mu, \ldots\right. \\
& \left.\gamma_{1} \gamma_{2}+\gamma_{2}\left(a+b \lambda_{p}+c \lambda_{p}\right)+\gamma_{3} \gamma_{4}+\gamma_{4} \mu\right)
\end{aligned}
$$

where $\mu$ is the sum of the elements in the first row of $A$ and $\lambda_{p}=\frac{p-1}{2}$.

$$
\begin{aligned}
-B_{2}^{T} B_{1}^{T}+Q B_{2}^{T}+B_{4}^{T} B_{3}^{T}+A B_{4}^{T} & =\left(\begin{array}{c}
\gamma_{2} \\
\vdots \\
\gamma_{2}
\end{array}\right) \gamma_{1}+Q\left(\begin{array}{c}
\gamma_{2} \\
\vdots \\
\gamma_{2}
\end{array}\right)+\left(\begin{array}{c}
\gamma_{4} \\
\vdots \\
\gamma_{4}
\end{array}\right) \gamma_{3}+A\left(\begin{array}{c}
\gamma_{4} \\
\vdots \\
\gamma_{4}
\end{array}\right) \\
& =\left(\begin{array}{c}
\gamma_{1} \gamma_{2} \\
\vdots \\
\gamma_{1} \gamma_{2}
\end{array}\right)+\left(\begin{array}{c}
\gamma_{2}\left(a+b \lambda_{p}+c \lambda_{p}\right) \\
\vdots \\
\gamma_{2}\left(a+b \lambda_{p}+c \lambda_{p}\right)
\end{array}\right)+\left(\begin{array}{c}
\gamma_{3} \gamma_{4} \\
\vdots \\
\gamma_{3} \gamma_{4}
\end{array}\right)+\left(\begin{array}{c}
\mu \gamma_{4} \\
\vdots \\
\mu \gamma_{4}
\end{array}\right) \\
& =\left(\begin{array}{c}
\gamma_{1} \gamma_{2}+\gamma_{2}\left(a+b \lambda_{p}+c \lambda_{p}\right)+\gamma_{3} \gamma_{4}+\mu \gamma_{4} \\
\vdots \\
\gamma_{1} \gamma_{2}+\gamma_{2}\left(a+b \lambda_{p}+c \lambda_{p}\right)+\gamma_{3} \gamma_{4}+\mu \gamma_{4}
\end{array}\right) .
\end{aligned}
$$




$$
\begin{aligned}
\text { - } B_{2}^{T} B_{2}+Q Q^{T}+B_{4}^{T} B_{4}+A A^{T} & =\left(\begin{array}{c}
\gamma_{2} \\
\vdots \\
\gamma_{2}
\end{array}\right)\left(\gamma_{2}, \ldots, \gamma_{2}\right)+Q Q^{T}+\left(\begin{array}{c}
\gamma_{4} \\
\vdots \\
\gamma_{4}
\end{array}\right)\left(\gamma_{4}, \ldots, \gamma_{4}\right)+A A^{T} \\
& =\left(\gamma_{2}+\gamma_{4}\right)^{2}\left(\begin{array}{ccc}
1 & \cdots & 1 \\
\vdots & \ddots & \vdots \\
1 & \cdots & 1
\end{array}\right)+Q Q^{T}+A A^{T} .
\end{aligned}
$$

Using (2) for $Q Q^{T}$, we see that

$$
B_{2}^{T} B_{2}+Q Q^{T}+B_{4}^{T} B_{4}+A A^{T}=\left\{\begin{array}{lr}
Q_{p}\left(\left(\gamma_{2}+\gamma_{4}\right)^{2}+a^{2},\left(\gamma_{2}+\gamma_{4}\right)^{2}+b^{2}+k(b+c)^{2},\left(\gamma_{2}+\gamma_{4}\right)^{2}\right. & \\
\left.+c^{2}+k(b+c)^{2}\right)+A A^{T} & \text { if } p=4 k+1 \\
Q_{p}\left(\left(\gamma_{2}+\gamma_{4}\right)^{2}+a^{2}+b^{2}+c^{2},\left(\gamma_{2}+\gamma_{4}\right)^{2}+a b+a c\right. & \\
\left.+k\left(b^{2}+c^{2}\right)+b c,\left(\gamma_{2}+\gamma_{4}\right)^{2}+a b+a c+k\left(b^{2}+c^{2}\right)+b c\right)+A A^{T} & \text { if } p=4 k+3
\end{array}\right.
$$

Combining all these results and factoring in the characteristic of the ambient ring, we arrive at the following results:

Theorem 3.2. Assume that $p=4 k+1$. Then, $C$ is a self-orthogonal code if and only if the following conditions hold:

(1) $\sum_{i=1}^{4} \gamma_{i}=0$,

(2) $\gamma_{1} \gamma_{2}+\gamma_{2} a+\gamma_{3} \gamma_{4}+\gamma_{4} \mu=0$

(3) $A A^{T}=Q_{p}\left(\left(\gamma_{2}+\gamma_{4}\right)^{2}+a^{2},\left(\gamma_{2}+\gamma_{4}\right)^{2}+b^{2}+k(b+c)^{2},\left(\gamma_{2}+\gamma_{4}\right)^{2}+c^{2}+k(b+c)^{2}\right)$.

Theorem 3.3. Assume that $p=4 k+3$. Then, $C$ is a self-orthogonal code if and only if the following conditions hold:

(1) $\sum_{i=1}^{4} \gamma_{i}=0$,

(2) $\gamma_{1} \gamma_{2}+\gamma_{2}(a+b+c)+\gamma_{3} \gamma_{4}+\mu \gamma_{4}=0$,

(3) $A A^{T}=Q_{p}\left(\left(\gamma_{2}+\gamma_{4}\right)^{2}+a^{2}+b^{2}+c^{2},\left(\gamma_{2}+\gamma_{4}\right)^{2}+a b+a c+k\left(b^{2}+c^{2}\right)+b c,\left(\gamma_{2}+\gamma_{4}\right)^{2}+a b+\right.$ $\left.a c+k\left(b^{2}+c^{2}\right)+b c\right)$.

\section{NuMERICAL RESULTS}

We will apply the construction methods described in section 3 to obtain extremal binary self-dual codes of various lengths. We need to point out that the theorems in section 3 provide us with necessary and sufficient conditions for when the codes generated are self-orthogonal. This is one of the points of distinction that our constructions have from those introduced in [7]. Using a random circulant matrix $A$ instead of $I_{n}$ means that the rank of the codes might not always be that of a self-dual code. However, we believe using a random circulant matrix brings more variety and helps us find more self-dual codes as has been demonstrated by our computations. All the computations are run on Magma ([2]).

4.1. New Codes of lengths 64. The possible weight enumerators for a self-dual Type I [64, 32, 12]code is given in $[4,5]$ as:

$$
\begin{aligned}
& W_{64,1}=1+(1312+16 \beta) y^{12}+(22016-64 \beta) y^{14}+\cdots, 14 \leq \beta \leq 284, \\
& W_{64,2}=1+(1312+16 \beta) y^{12}+(23040-64 \beta) y^{14}+\cdots, 0 \leq \beta \leq 277
\end{aligned}
$$

With the most updated information, [1], extremal singly even self-dual codes with weight enumerator $W_{64,1}$ are known

$$
\beta \in\left\{\begin{array}{c}
14,16,18,20,22,24,25,26,28,29,30,32, \\
34,35,36,38,39,44,46,53,59,60,64,74
\end{array}\right\}
$$


and extremal singly even self-dual codes with weight enumerator $W_{64,2}$ are known for

$$
\beta \in\left\{\begin{array}{l}
0,1, \ldots, 42,44,45,48,50,51,52,56,58,64,65 \\
72,80,88,96,104,108,112,114,118,120,184
\end{array}\right\} \backslash\{31,39\} .
$$

In this section, we obtain the codes with weight enumerators for $\beta=48$ and 50 in $W_{64,1}$. We apply Theorem 3.3 for $p=7$ over $\mathbb{F}_{4}+u \mathbb{F}_{4}$ and obtain Type I codes of length 64 as binary images.

TABLE 1. Self-dual codes over $\mathbb{F}_{4}+u \mathbb{F}_{4}$ when $p=7$

\begin{tabular}{cccc|c|c}
\hline$C_{i}$ & $\left(\gamma_{1}, \gamma_{2}, \gamma_{3}, \gamma_{4}\right)$ & $(a, b, c)$ & $r_{A}$ & Aut $\left(C_{i}\right)$ & $W_{64,1}$ \\
\hline 1 & $(0,1, B, A)$ & $(B, 4, F)$ & $(A, 4,6,5, E, 7, F)$ & $2^{2} \cdot 7$ & $\beta=18$ \\
\hline 2 & $(0,1, B, A)$ & $(1,6,7)$ & $(A, 4,6, F, E, 5,7)$ & $2^{2} \cdot 7$ & $\beta=32$ \\
\hline 3 & $(0,1, B, A)$ & $(1,6,7)$ & $(A, 4,6,7, E, F, 5)$ & $2^{2} \cdot 7$ & $\beta=46$ \\
\hline 4 & $(0,1, B, A)$ & $(1,6,7)$ & $(A, 4,6,5, E, 7, F)$ & $2^{2} \cdot 7$ & $\beta=60$ \\
\hline
\end{tabular}

Self dual binary codes $C$ and $D$ of length $n$ are said to be neighbors if $\operatorname{dim}(C \cap D)=n / 2-1$. In order to consider some neighbors of a code $C$ we pick a vector $x \in \mathbb{F}_{2}^{n}-C$ and let $D=\left\langle\langle x\rangle^{\perp} \cap C, x\right\rangle$. We use the standard form of the generator matrix of $C$, which lets us to fix first $n / 2$ entries of $x$ without loss of generality. We set the first 32 entries of $x$ to be 0 . We consider the neighbours of the binary images of the codes in Table 1 and obtain new codes of length 64 which are listed in Table 2.

TABle 2. New codes of length $64\left(W_{64,1}\right)$ as neighbours of codes in Table 1

\begin{tabular}{ccccc}
\hline $\mathcal{D}_{64, i}$ & $C_{j}$ & $\left(x_{33}, x_{34}, \ldots, x_{64}\right)$ & $\beta$ & $\left|A u t\left(\mathcal{D}_{64, i}\right)\right|$ \\
\hline 1 & 4 & $(00001100111100110111000000001110)$ & $\mathbf{4 8}$ & 2 \\
\hline 2 & 4 & $(01110111100100101111100100110111)$ & $\mathbf{5 0}$ & 2 \\
\hline
\end{tabular}

4.2. New extremal self-dual codes of lengths 66. The weight enumerator of an extremal selfdual code of length 66 is given in [5] as follows:

$$
\begin{aligned}
& W_{66,1}=1+(858+8 \beta) y^{12}+(18678-24 \beta) y^{14}+\cdots \quad \text { where } 0 \leq \beta \leq 778, \\
& W_{66,2}=1+1690 y^{12}+7990 y^{14}+\cdots \text { and } \\
& W_{66,3}=1+(858+8 \beta) y^{12}+(18166-24 \beta) y^{14}+\cdots \text { where } 14 \leq \beta \leq 756 .
\end{aligned}
$$

Together with the codes recently obtained in [1] and the ones from [12], [14], [15] and [16], extremal singly even self-dual codes with weight enumerator $W_{66,1}$ are known for

$$
\beta \in\{0,1,2,3,5,6, \ldots, 94,100,101,115\}
$$

and extremal singly even self-dual codes with weight enumerator $W_{66,3}$ are known for

$$
\beta \in\{22,23, \ldots, 92\} \backslash\{65,68,69,72,89,91\} .
$$

In this section, we construct the codes with weigth enumerators for $\beta=65,68,69$ and 72 in $W_{66,3}$ as extensions of codes in Section 4.1.

We recall the following extension theorem that helps us construct self-dual codes of length $2 n+2$ from self-dual codes of length $2 n$ :

Theorem 4.1. ([17]) Let $C$ be a binary self-dual code of length $2 n, G=\left(r_{i}\right)$ be an $n \times 2 n$ generator matrix for $C$, where $r_{i}$ is the $i$-th row of $G, 1 \leq i \leq n$. Let $X$ be a vector in $\mathbb{F}_{2}^{2 n}$ with $\langle X, X\rangle=1$. Let $y_{i}=\left\langle r_{i}, X\right\rangle$ for $1 \leq i \leq n$. Then the following matrix

$$
\left[\begin{array}{cc|c}
1 & 0 & X \\
\hline y_{1} & y_{1} & r_{1} \\
\vdots & \vdots & \vdots \\
y_{n} & y_{n} & r_{n}
\end{array}\right]
$$


generates a binary self-dual code of length $2 n+2$.

We apply Theorem 4.1 to the codes of length 64 tabulated in Table 1 and 2 to find extremal self-dual codes of length 66 , with $X=\left(0_{32} \mid x\right)$, where $0_{32}$ denotes that the first 32 bits of $X$ are 0 's. The new codes of length 66 that we obtain are tabulated in the following table:

TABLE 3. Extremal Self-dual code of length 66 from binary extensions of codes of length 64

\begin{tabular}{ccccc}
\hline $\mathcal{N}_{66, i}$ & Code & $x$ & $W_{66,3}$ & $\mid$ Aut $\left(\mathcal{N}_{66, i}\right) \mid$ \\
\hline 1 & $C_{3}$ & $(10101101111000010010011001101101)$ & $\beta=\mathbf{6 5}$ & 1 \\
\hline 2 & $\mathcal{D}_{64,2}$ & $(11010111011111000001101111100100)$ & $\beta=\mathbf{6 8}$ & 1 \\
\hline 3 & $C_{3}$ & $(00011111011010110011100110110000)$ & $\beta=\mathbf{6 9}$ & 1 \\
\hline 4 & $C_{4}$ & $(00001111111011001101011110101001)$ & $\beta=\mathbf{7 2}$ & 1 \\
\hline
\end{tabular}

4.3. New extremal self-dual codes of length 68. The possible weight enumerator of an extremal binary self-dual code of length 68 (of parameters $[68,34,12]$ ) is in one of the following forms by $[3,10]$ :

$$
\begin{aligned}
& W_{68,1}=1+(442+4 \beta) y^{12}+(10864-8 \beta) y^{14}+\cdots, 104 \leq \beta \leq 1358 \\
& W_{68,2}=1+(442+4 \beta) y^{12}+(14960-8 \beta-256 \gamma) y^{14}+\cdots
\end{aligned}
$$

where $0 \leq \gamma \leq 9$. Recently, Yankov et al. constructed the first examples of codes with a weight enumerator for $\gamma=7$ in $W_{68,2}$. The new codes we have constructeed all have $\gamma=0,1$ or 2 in $W_{68,2}$. So, in order to save space we only give the list of known values for $\gamma=0, \gamma=1$ and $\gamma=2$ in $W_{68,2}$. For a full list of known codes we refer to [9].

$$
\begin{aligned}
& \gamma=0, \beta=0,7,11,14,17,21,22,28,33,35,42,44, \ldots, 158,159,161,163,165 \\
& \quad 175,187,189,203,209,221,231,255,303 \text { or } \\
& \beta \in\{2 m \mid m=17,20,102,110,119,136,165 \text { or } 80 \leq m \leq 99\} \\
& \gamma=1, \beta=49,51,53,55,57,59, \ldots, 160,161,163,165,167,169,171 \text { or } \\
& \beta \in\{2 m \mid m=22,24, \ldots, 29,81, \ldots, 90,92, \ldots, 96\} ; \\
& \gamma=2, \beta=58,65,69,71,73,75,77,79,81,157,159,206,208 \text { or } \beta \in\{2 m \mid 30 \leq m \leq 100\} \text { or } \\
& \beta \in\{2 m+1 \mid 41 \leq m \leq 77\}
\end{aligned}
$$

In the following table, we list self-dual codes of length 68 as Gray images of codes over $\mathbb{F}_{2}+u \mathbb{F}_{2}$ via Theorem 3.1 for $p=17$.

TABLE 4. Self-dual codes of length 68 via $\mathbb{F}_{2}+u \mathbb{F}_{2}$ when $p=17\left(W_{68,2}\right)$

\begin{tabular}{ccc|c|cc}
\hline $\mathcal{D}_{i}$ & $(a, b, c)$ & $r_{A}$ & $A u t\left(\mathcal{D}_{i}\right)$ & $\alpha$ & $\beta$ \\
\hline 1 & $(1, u, u)$ & $(0,0,0, u, u, 1,3,3,0, u, 1,3, u, 3,3,1,3)$ & $2^{2} \cdot 7$ & 0 & $\beta=204$ \\
\hline 2 & $(1, u, u)$ & $(u, u, u, 0,0,3,3,3,0, u, 1,3,0,3,3,1,1)$ & $2^{2} \cdot 7$ & 0 & $\beta=238$ \\
\hline 3 & $(1, u, u)$ & $(0, u, 0,0,0,1,3,1,0, u, 3,3,0,1,1,3,3)$ & $2^{2} \cdot 7$ & 0 & $\beta=272$ \\
\hline
\end{tabular}

By considering the neighbours of codes in Table 4 we construct 14 new codes of length 68 , which are listed in Table 5. The generator matrix of $\mathcal{D}_{j}$ is used in standard form. Therefore, we assume the first 34 entries to be 0 without loss of generality. 
TABLE 5. New codes of length $68\left(W_{68,2}\right)$ as neighbours of codes in Table 4

\begin{tabular}{cccccc}
\hline $\mathcal{N}_{68, i}$ & $\mathcal{D}_{j}$ & $\left(x_{35}, x_{36}, \ldots, x_{68}\right)$ & $\gamma$ & $\beta$ & $\left|A u t\left(\mathcal{N}_{68, i}\right)\right|$ \\
\hline 1 & 1 & $(1110111011010001001101010001000010)$ & 0 & $\mathbf{1 6 7}$ & 2 \\
\hline 2 & 3 & $(1010111000110100000100110101100110)$ & 0 & $\mathbf{1 6 9}$ & 2 \\
\hline 3 & 2 & $(1111001010000110011001100100001000)$ & 0 & $\mathbf{1 7 1}$ & 1 \\
\hline 4 & 3 & $(0010001101101100001011101001111110)$ & 0 & $\mathbf{1 7 3}$ & 1 \\
\hline 5 & 2 & $(1101000110101100010001001011110111)$ & 0 & $\mathbf{1 7 7}$ & 1 \\
\hline 6 & 2 & $(1001000000100110010011100110010011)$ & 0 & $\mathbf{1 7 9}$ & 1 \\
\hline 7 & 3 & $(0001100001110011001101010011001110)$ & 0 & $\mathbf{1 8 1}$ & 2 \\
\hline 8 & 3 & $(1001101101101001110101000100111100)$ & 0 & $\mathbf{2 0 2}$ & 2 \\
\hline 9 & 3 & $(1001011000110010011010011010110010)$ & 0 & $\mathbf{2 1 7}$ & 2 \\
\hline 10 & 1 & $(1110110110110001110001000000001101)$ & 1 & $\mathbf{1 8 3}$ & 2 \\
\hline 11 & 3 & $(0110011100110101010110111101011111)$ & 1 & $\mathbf{1 9 9}$ & 2 \\
\hline 12 & 3 & $(0100001000110111001011000001100000)$ & 1 & $\mathbf{2 0 7}$ & 1 \\
\hline 13 & 3 & $(1100111101100001111011110011110000)$ & 1 & $\mathbf{2 1 4}$ & 2 \\
\hline 14 & 2 & $(1011101010010000110010110000100111)$ & 2 & $\mathbf{2 1 6}$ & 2 \\
\hline
\end{tabular}

\section{Conclusion}

In this work, we introduced modified versions of quadratic double circulant and bordered quadratic double circulant constructions for self-dual codes. We demonstrated the relevance of this new construction by constructing many binary self-dual codes, including new extremal binary self-dual codes of length 64,66 and 68 .

- Codes of length 64: We were able to construct the following extremal binary self-dual codes with new weight enumerators in $W_{64,1}$ :

$$
\beta=\{48,50\} \text {. }
$$

- Codes of length 66: We were able to construct the following extremal binary self-dual codes with new weight enumerators in $W_{66,3}$ :

$$
\beta=\{65,68,69,72\} .
$$

- Codes of length 68: We were able to construct the following extremal binary self-dual codes with new weight enumerators in $W_{68,2}$ :

$$
\begin{aligned}
& (\gamma=0, \quad \beta=\{167,169,171,173,177,179,181,202,217\}) . \\
& (\gamma=1, \quad \beta=\{183,199,207,214\}) . \\
& (\gamma=2, \quad \beta=\{216\}) .
\end{aligned}
$$

A possible direction for future research could be considering different primes $p$ and different alphabets that could result in self-dual codes of different lengths.

Acknowledgment: The authors would like to thank the anonymous referees and the editor for their valuable remarks that improved the presentation of this paper.

\section{REFERENCES}

[1] D. Anev, M. Harada, and N. Yankov, New extremal singly even self-dual codes of lengths 64 and 66, J. Algebra Comb. Discrete Struct. Appl. 5 (2018), no. 3, 143-151.

[2] W. Bosma, J. Cannon, and C. Playoust, The Magma algebra system. I. The user language, J. Symbolic Comput. 24 (1997), no. 3-4, 235-265. Computational algebra and number theory (London, 1993).

[3] S. Buyuklieva and I. Boukliev, Extremal self-dual codes with an automorphism of order 2, IEEE Trans. Inform. Theory 44 (1998), no. 1, 323-328.

[4] J. H. Conway and Sloane N.J.A, A new upper bound on the minimal distance of self-dual codes, IEEE Trans. Inform. Theory 36 (1990), no. 6, 1319-1333.

[5] S.T. Dougherty, T.A. Gulliver, and M. Harada, Extremal binary self-dual codes, IEEE Trans. Inform. Theory 43 (1997), no. 6, 2036-2047. 
[6] S.T. Dougherty, P. Gaborit, M. Harada, and Patrick Solé, Type II codes over $\mathbf{F}_{2}+u \mathbf{F}_{2}$, IEEE Trans. Inform. Theory 45 (1999), no. 1, 32-45.

[7] P. Gaborit, Quadratic double circulant codes over fields, J. Combin. Theory Ser. A 97 (2002), no. 1, 85-107.

[8] J. Gildea, H. Hamilton, A. Kaya, and B. Yildiz, "Binary generator matrices for extremal binary self-dual codes of lengths 64,66 and 68", available at http://abidinkaya.wixsite.com/math/gildeaqr.

[9] J. Gildea, A. Kaya, and B. Yildiz, An altered four circulant construction for self-dual codes from group rings and new extremal binary self-dual codes I, Discrete Math. 342 (2019), no. 12, 111620, 8.

[10] M. Harada and A. Munemasa, Some restrictions on weight enumerators of singly even self-dual codes, IEEE Trans. Inform. Theory 52 (2006), no. 3, 1266-1269.

[11] M. Harada, New quantum codes constructed from some self-dual additive $F_{4}$-codes, Inform. Prcoess. Letters 138 (2018), 35-38.

[12] S. Karadeniz and B. Yildiz, New extremal binary self-dual codes of length 66 as extensions of self-dual codes over $R_{k}$, J. Franklin Inst. 350 (2013), no. 8, 1963-1973.

[13] A. Kaya, B. Yildiz, and I. Siap, Quadratic residue codes over $\mathbb{F}_{p}+v \mathbb{F}_{p}$ and their Gray images, J. Pure Appl. Algebra 218 (2014), no. 11, 1999-2011.

[14] A. Kaya, New extremal binary self-dual codes of lengths 64 and 66 from $R_{2}$-lifts, Finite Fields Appl. 46 (2017), $271-279$.

[15] A. Kaya, B. Yildiz, and A. Pasa, New extremal binary self-dual codes from a modified four circulant construction, Discrete Math. 339 (2016), no. 3, 1086-1094.

[16] A. Kaya, B. Yildiz, and I. Siap, New extremal binary self-dual codes from $\mathbb{F}_{4}+u \mathbb{F}_{4}$-lifts of quadratic circulant codes over $\mathbb{F}_{4}$, Finite Fields Appl. 35 (2015), 318-329.

[17] J. L. Kim, New extremal self-dual codes of lengths 36, 38, and 58, IEEE Trans. Inform. Theory 47 (2001), no. 1, 386-393.

[18] S. Ling and P. Solé, Type II codes over $\mathbf{F}_{4}+u \mathbf{F}_{4}$, European J. Combin. 22 (2001), no. 7, 983-997.

[19] E.M. Rains, Shadow bounds for self-dual codes, IEEE Trans. Inform. Theory 44 (1998), no. 1, $134-139$.

[20] M.Shi, L. Qian, Y. Liu, and P. Solé, Good self-dual generalized quasi-cylic codes exist, Inform. Process. Letters 118 (2017), 21-24.

[21] M.Shi, L. Qian, L. Sok, and P. Solé, On constacyclic codes over $\left.\mathbb{Z}_{4}[u] /<u^{2}-1\right\rangle$ and their Gray images, Finite Fields Appl. 45 (2017), 86-95.

[22] M.Shi, Y. Guan, and P. Solé, Two new families of two-weight codes, IEEE Trans. Inform. Theory 63 (2017), no. $10,6240-6246$.

Department of Mathematics, Faculty of Science and Engineering, University of Chester, England Email address: j.gildea@chester.ac.uk

Department of Mathematics, Faculty of Science and Engineering, University of Chester, England Email address: holham30@gmail.com

Department of Mathematics Education, Sampoerna University, 12780, Jakarta, Indonesia

Email address: abidin.kaya@sampoernauniversity.ac.id

Department of Mathematics \& Statistics, Northern Arizona University, Flagstaff, AZ 86001, USA

Email address: bahattin.yildiz@nau.edu 